\title{
Typ 2 Diabetes mellitus - Screening und Prävention
}

\section{Update 2016}

\author{
Marietta Stadler · Elke Fröhlich-Reiterer · Rudolf Prager
}

Online publiziert: 6. April 2016

(C) Die Autor(en) 2016. Dieser Artikel ist auf Springerlink.com mit Open Access verfügbar.

Zusammenfassung Die Diabetesprävalenz nimmt weltweit $\mathrm{zu}$, wobei etwa die Hälfte aller Betroffenen noch nicht diagnostiziert ist. Dieses Positionspapier der Österreichischen Diabetesgesellschaft basiert auf aktueller Evidenz zu Screening und Prävention des Typ 2 Diabetes mellitus.

Schlüsselwörter Diabetesprävention · Typ 2 Diabetes · Diabetesscreening · Leitlinie

Type 2 Diabetes mellitus-screening and prevention - Update 2016

Summary The prevalence of diabetes is increasing in westernized countries. In addition, about half of all patients suffering from diabetes are not diagnosed. The current article represents the recommendations of the Austrian Diabetes Association for the screening and prevention of type 2 diabetes, based on currently available evidence.

\section{Stadler ( $\square)$}

Diabetes Research Group, King's College London,

Großbritannien,

London, England

E-Mail: marietta.stadler@kcl.ac.at

R. Prager

3. Medizinische Abteilung für Stoffwechselerkrankungen

und Nephrologie und Karl-Landsteiner-Institut für

Stoffwechselerkrankungen und Nephrologie,

Krankenhaus Wien-Hietzing,

Wien, Österreich

E-Mail: rudolf.prager@wienkav.at

E. Fröhlich-Reiterer

Klinische Abteilung für allgemeine Pädiatrie,

Medizinische Universität Graz,

Graz, Österreich
Keywords Diabetes prevention . Type 2 diabetes . Diabetes screening · Guideline

\section{Epidemiologie des Typ 2 Diabetes mellitus}

Die weltweite Prävalenz von Typ 2 Diabetes mellitus (T2DM) bei Erwachsenen wurde im Jahr 2000 auf 151 Mio. geschätzt, wobei eine Zunahme um $46 \%$ auf 221 Mio. bis 2010 und 300 Mio. bis zum Jahr 2025 angenommen wurde [1, 2]. Im Jahr 2013 wurden diese Zahlen nach oben revidiert: 2013 waren 382 Mio. an Diabetes erkrankt, und die Schätzung für 2035 beläuft sich auf 592 Mio. Diabetiker weltweit. Die größte Zunahme wird in Ländern mit niedrigem und mittlerem Einkommensniveau erwartet [3].

In Österreich wird gemäß einer Erhebung der Statistik Austria die Diabetesprävalenz auf etwa $6 \%$ geschätzt (Statistik Austria, Gesundheitsbefragung 2006/2007). Ausgehend von epidemiologischen Studien sind jedoch etwa die Hälfte der Typ 2 Diabetiker noch nicht als solche diagnostiziert [4], haben aber bereits ein erhöhtes Risiko für Schlaganfall, Herzinfarkt und periphere arterielle Verschlusskrankheit [5, 6]. Angesichts der DiabetesPandemie mit deren gesundheitlichen und sozioökonomischen Konsequenzen sind effiziente Strategien $\mathrm{zu}$ Prävention und Früherkennung des T2DM erforderlich.

\section{Risikofaktoren für T2DM}

Ursachen des T2DM sind hauptsächlich auf Lebensstil-bedingte Faktoren, wie Bewegungsmangel und hyperkalorische, fettreiche Ernährung sowie auf eine genetische Disposition zurückzuführen. Das Risiko, einen T2DM zu entwickeln, steigt mit Lebensalter, Übergewicht und Bewegungsarmut an. Nachkommen oder Geschwister von Typ 2 Diabetikern sowie Frauen 
nach Schwangerschaftsdiabetes (Gestationsdiabetes) weisen ebenfalls ein erhöhtes T2DM-Risiko auf, ebenso Personen asiatischer, afrikanischer oder lateinamerikanischer Herkunft [7].

Patienten mit metabolischem Syndrom (Insulinresistenzsyndrom) mit den Teilkomponenten Glukosestoffwechselstörung (IGT), Adipositas, Dyslipidämie und/oder arterielle Hypertonie stellen die Hauptrisikogruppe für die Entwicklung eines Typ 2 Diabetes dar, und sind deshalb auch die primäre Zielgruppe für Screening und Diabetesprävention.

\section{T2DM-Screening und Prävention - aktuelle Empfehlungen}

\section{Systematisches Screening auf T2DM}

Personen mit erhöhtem Diabetesrisiko sollten identifiziert und systematisch auf das Vorliegen von T2DM oder Prädiabetes (gestörte Glukosetoleranz; IGT und/ oder gestörte Nüchternglukose; IFG) gescreent werden (Empfehlungsgrad B).

Die Nüchternplasmaglukose, alternativ HbAlc oder oraler Glukosetoleranztest, sollte ab dem Lebensalter von 45 Jahren in 3-jährigem Abstand kontrolliert werden (E). Wenn zusätzlich einer der in Tab. 1 aufgelisteten weiteren Risikofaktoren vorliegt, soll häufiger und auch bei jüngeren Personen gescreent werden [7] (Tab. 2). Bei einem Nüchternblutzucker $>100 \mathrm{mg} / \mathrm{dl}$ sollte ein oraler Glukosetoleranztest durchgeführt werden [7] (Empfehlungsgrad B).

\section{Lebensstilmodifikation}

Lebensstilmodifikation mit gesunder Ernährung, Gewichtsreduktion und körperlicher Aktivität kann nicht nur Entstehen von T2DM verhindern oder verzögern, sondern wirkt sich insgesamt günstig auf das kardiovaskuläres Risiko und die Lebensqualität aus. Die
Tab. 2 Kriterien für Typ-2-Diabetesscreening bei Kindern und Jugendlichen. (Adaptiert nach [8])

Kriterien für Typ-2-Diabetesscreening bei Kindern und Jugendlichen

Ab dem 10. Lebensjahr bei Übergewicht (BMI $>90$.

Perzentile) undVorliegen vonmindestens zweider folgenden Risikofaktoren

Typ 2 Diabetes bei Verwandten 1. bis 2. Grades

Extreme Adipositas (BMl>99,5. Perzentile)

Zeichen der Insulinresistenz oder mit ihr assoziierte Veränderungen (arterieller Hyertonus, Dyslipidämie, erhöhte Transaminasen, polyzystisches Ovarialsyndrom, Acanthosis nigricans)

Zugehörigkeit zu einer Gruppe mit erhöhtem Risiko (z. B. Ostasiaten, Afroamerikaner, Hispanier)

BMI Body Mass Index

Diabetes Prevention-Study (DPS) [9] und das Diabetes Prevention Program (DPP) [10] dokumentieren bei Patienten mit IGT eine 58-prozentige relative Risikoreduktion für das Auftreten von T2DM durch Lebensstilmodifikation. In den Nachbeobachtungen beider Studien blieb das Risiko an T2DM zu erkranken, in den Interventionsgruppen anhaltend niedriger (34-36\% relative Risikoreduktion) $[11,12]$ und die LebensstilInterventionen erwiesen sich, obwohl aufwendig, als kosteneffektiv aus gesundheitsökonomischer Sicht [13].

Daher ist es wichtig, bei Personen mit erhöhtem T2DM-Risiko - selbst, wenn noch keine manifeste Glukosestoffwechselstörung vorliegt - Bewusstsein für die Bedeutung von Gewichtsreduktion und regelmäßiger körperlicher Aktivität zu schaffen. Zusätzliche kardiovaskuläre Risikofaktoren (z. B. Rauchen, Hypertonie und Dyslipidämie) sollen rechtzeitig erkannt und behandelt werden.

Wird beim Screening Prädiabetes festgestellt, sollte eine Lebensstil-Intervention in Form von strukturierter Beratung zu Ernährung und Bewegung und regelmäßigen Follow-ups vorgenommen werden, um eine Körpergewichtsabnahme von ca. $7 \%$ und $150 \mathrm{~min} /$ Woche körperliche Aktivität zu erzielen (Empfehlungs$\operatorname{grad} \mathrm{B})$.

Tab. 1 Kriterien für Diabetesscreening bei asymptomatischen Erwachsenen. (Adaptiert nach [1])

\begin{tabular}{l} 
Kriterien für Diabetesscreening bei asymptomatischen Erwachsenen \\
Bei Alter $\geq 45$ Jahre \\
\hline Unabhängig vom Alter bei Übergewicht $\left(\mathrm{BMI} \geq 25 \mathrm{~kg} / \mathrm{m}^{2}\right.$ ) und einem oder mehreren zusätzlichen Risikofaktoren \\
\hline Physische Inaktivität \\
\hline Verwandte ersten Grades mit Diabetes \\
Arterielle Hypertonie ( $\geq 140 / 90 \mathrm{mmHg}$ oder antihypertensive Therapie) \\
\hline HDL Cholesterin Männer $<35 \mathrm{mg} / \mathrm{dl}$ und/oder Triglyceride $>250 \mathrm{mg} / \mathrm{dl}$ \\
\hline Polyzystisches Ovarialsyndrom, Geburt eines Kindes mit $>4,5 \mathrm{~kg}$ Körpergewicht, oder vorangegangenem Schwangerschaftsdiabetes \\
\hline IFG oder IGT zu einem früheren Zeitpunkt, HbA1c $\geq 5,7$ \\
\hline Kardio- oder zerebrovaskuläre Erkrankung \\
\hline Hochrisikopopulation (asiatische, afrikanische, lateinamerikanische Herkunft) \\
\hline Acanthosis nigricans \\
\hline BMI Body Mass Index, IFG gestörte Nüchternglukose, IGT gestörte Glukosetoleranz \\
\hline
\end{tabular}




\section{Ernährung}

Grundsätzlich sollte die Ernährung auf Basis einer gesunden Mischkost erfolgen, die fettarm, kohlenhydrat- und ballaststoffreich ist. Weniger als $30 \%$ des Tagensenergiebedarfs soll durch Fett, weniger als $10 \%$ durch gesättigte Fettsäuren gedeckt werden. Übergewichtige sollen Gewichtsreduktion von ca. 5-10\% des Körpergewichtes durch kalorienreduzierte Diät und körperliche Aktivität erreichen. Bei arterieller Hypertonie sollte die Nahrung kochsalzarm, bei Hyperlipidämie cholesterin- und fettarm und bei Hyperurikämie purinarm sein [1].

\section{Körperliche Aktivität}

Gemäß den Empfehlungen der Amerikanischen Diabetesgesellschaft (ADA), sollten Personen mit erhöhtem Diabetesrisiko, ebenso wie Patienten mit Prädiabetes oder manifestem T2DM, zu regelmäßiger moderater körperliche Aktivität (30 min/Tag, bzw. $150 \mathrm{~min} /$ Woche) motiviert werden [7].

\section{Medikamentöse Diabetesprävention}

Als MedikamentemitPotenzialfürdieDiabetesprävention haben sich Metformin, alpha-Glukosidasehemmer, Orlistat und Glitazone herauskristallisiert. Keine der medikamentösen Interventionen war jedoch so effektiv wie die Lebensstilintervention. Metformin ist die Substanz mit der besten Evidenzlage hinsichtlich Effektivität, Langzeitsicherheit und Kosteneffizienz [13, 14].

Bei besonders hohem Diabetesrisiko (IFG, IGT, oder einem $\mathrm{HbAlc}>5,7 \%$, insbesondere jene mit Adipositas $>35 \mathrm{~kg} / \mathrm{m} 2$, Alter $<60$ Jahren oder mit vorangegangenem Gestationsdiabetes) kann daher auch die Gabe von Metformin erwägt werden [7], (Empfehlungs$\operatorname{grad} \mathrm{A})$.

Abschließend sei die enorme Bedeutung von Screening und Prävention bei T2DM, einer Erkrankung mit rapid steigender Inzidenz, hervorgehoben. Lebensstilmodifikation mit Diät und körperlicher Aktivität ist die effektivste Maßnahme zur Prävention des T2DM.

\section{Einhaltung ethischer Richtlinien}

\section{Interessenkonflikt}

M. Stadler hat in den letzten 36 Monaten keine Forschungsunterstützungen und/oder Honorare von Unternehmen erhalten.

E. Fröhlich-Reiterer hat in den letzten 36 Monaten von folgenden Firmen Honorare/Forschungsgelder erhalten: Eli Lilly, Medtronic, Novo Nordisk, sanofi-aventis, Roche.

R. Prager hat von folgenden Unternehmen, die auch fördernde Mitglieder der ÖDG sind, Forschungsunterstützungen und/oder Honorare erhalten: Abbott, AstraZeneca, Bristol-Myers Squibb, Johnson \& Johnson
Medical, Eli Lilly, Merck Sharp \& Dohme, Novartis, Novo Nordisk, Roche, sanofi-aventis, Takeda.

\section{Open Access}

Dieser Artikel unterliegt den Bedingungen der Creative Commons Attribution License. Dadurch sind die Nutzung, Verteilung und Reproduktion erlaubt, sofern der/die Originalautor/en und die Quelle angegeben sind.

\section{Literatur}

1. King H, Aubert RE, Herman WH. Global burden of diabetes, 1995-2025: prevalence, numerical estimates, and projections. Diabetes Care. 1998;21:1414-31.

2. Zimmet P, Alberti KG, Shaw J. Global and societal implications of the diabetes epidemic. Nature. 2001;414:782-7.

3. Guariguata L, Whiting DR, Hambleton I, Beagley J, Linnenkamp U, Shaw JE. Global estimates of diabetes prevalence for 2013 and projections for 2035. Diabetes Res Clin Pract. 2014;103:137-49.

4. Rathmann W, Haastert B, Icks A, Lowel H, Meisinger C, Holle R, Giani G. High prevalence of undiagnosed diabetes mellitus in Southern Germany: target populations for efficient screening. The KORA survey 2000. Diabetologia. 2003;46:182-9.

5. Rendell M, Kimmel DB, Bamisedun O, O'Donnell ET, Fulmer J. The health care status of the diabetic population as reflected by physician claims to a major insurer. Arch Intern Med. 1993;153:1360-6.

6. Rodriguez BL, Lau N, Burchfiel CM, Abbott RD, Sharp DS, Yano K, Curb JD. Glucose intolerance and 23-year risk of coronary heart disease and total mortality: the Honolulu Heart Program. Diabetes Care. 1999;22:1262-5.

7. Standards of medical care in diabetes - 2015: summary of revisions. Diabetes Care 2015;38 Suppl:S4.

8. Neu A, Beyer P, Burger-Busing J, Danne T, Etspuler J, Heidtmann B, Holl RW, Karges B, Kiess W, Knerr I, Kordonouri O, Lange K, Lepler R, Marg W, Nake A, Petersen M, Podeswik A, Stachow R, von Sengbusch S, Wagner V, Ziegler R, Holterhus PM. Diagnosis, therapy and control of diabetes mellitus in children and adolescents. Exp Clin Endocrinol Diabetes. 2014;122:425-34.

9. Tuomilehto J, Lindstrom J, Eriksson JG, Valle TT, Hamalainen H, Ilanne-Parikka P, Keinanen-Kiukaanniemi S, Laakso M, Louheranta A, Rastas M, Salminen V, Uusitupa M. Prevention of type 2 diabetes mellitus by changes in lifestyle among subjects with impaired glucose tolerance. $\mathrm{N}$ Engl J Med. 2001;344:1343-50.

10. Knowler WC, Barrett-Connor E, Fowler SE, Hamman RF, Lachin JM, Walker EA, Nathan DM. Reduction in the incidence of type 2 diabetes with lifestyle intervention or metformin. N Engl J Med. 2002;346:393-403.

11. Knowler WC, Fowler SE, Hamman RF, Christophi CA, Hoffman HJ, Brenneman AT, Brown-Friday JO, Goldberg R, Venditti E, Nathan DM. 10-year follow-up of diabetes incidence and weight loss in the Diabetes Prevention Program Outcomes Study. Lancet. 2009;374:1677-86. 


\section{leitlinien für die praxis}

12. Uusitupa M, Peltonen M, Lindstrom J, Aunola S, IlanneParikka P, Keinanen-Kiukaanniemi S, Valle TT, Eriksson JG, Tuomilehto J. Ten-year mortality and cardiovascular morbidity in the Finnish Diabetes Prevention Study secondary analysis of the randomized trial. PLoS One 2009;4:e5656.

13. Diabetes Prevention Program Research Group. The 10-year cost-effectiveness of lifestyle intervention or metformin for diabetes prevention: an intent-to-treat analysis of the DPP/ DPPOS. Diabetes Care. 2012;35:723-30.
14. Diabetes Prevention Program Research Group. Longterm safety tolerability, and weight loss associated with metformin in the Diabetes Prevention Program Outcomes Study. Diabetes Care. 2012;35:731-7. 